\title{
Modulation of Sensory Response at Different Time Lags after Locus Coeruleus Micro-stimulation
}

Zeinab Fazlali" \#, Yadollah Ranjbar-Slamloo ${ }^{1, \bullet}$, and Ehsan Arabzadeh²

1- School of Cognitive Sciences, Institute for Research in Fundamental Sciences (IPM), Tehran, Iran

2- Eccles Institute of Neuroscience, John Curtin School of Medical Research, The Australian National University, Canberra, ACT 2601, Australia

\# Current address: Department of Biomedical Engineering, Columbia University, New York, NY, USA

- Current address: Eccles Institute of Neuroscience, John Curtin School of Medical Research, The Australian National University, Canberra, ACT 2601, Australia

Number of Pages: 33

Number of Figures: 7

Number of words for Abstract: 262 words

Number of words for text: 4553 words

Keywords: Locus Coeruleus; neuromodulation; micro-stimulation; barrel cortex; sensory response.

\section{Corresponding Authors:}

Zeinab Fazlali, Current address: Department of Biomedical Engineering, Columbia University, New York, NY, USA

Email address: zeinab.fazlali@columbia.edu

Ehsan Arabzadeh, Eccles Institute of Neuroscience, John Curtin School of Medical Research, The Australian National University, Canberra, ACT 2601, Australia

Email address: ehsan.arabzadeh@anu.edu.au 


\begin{abstract}
Locus Coeruleus (LC) noradrenergic system has widespread projections throughout the brain and affects sensory processing in various modalities. Inhibition of both spontaneous and sensory-evoked cortical activity is well-documented in early experiments by either LC electrical micro-stimulation or local application of norepinephrine (NE). However, the temporal profile of LC modulation of sensory response is not well-established. Here, we recorded neuronal activity from the rat barrel cortex under urethane anesthesia and coupled LC micro-stimulation with brief mechanical deflection of whiskers at 10 different time lags (50-500 ms). LC micro-stimulation exhibited a biphasic effect on BC spontaneous activity: a period of suppression ( $\sim 100 \mathrm{~ms}$ ) followed by a period of excitation ( $200 \mathrm{~ms})$. This profile was highly consistent across BC units: $82 \%$ of BC units showed early suppression and 91\% of BC units showed late excitation. We observed a similar effect on the sensory-evoked response: at 50-ms lag, the evoked response (1-75 ms after deflection onset) decreased in $77 \%$ of units and at $150-\mathrm{ms} \mathrm{lag}$, the early evoked response was facilitated in $85 \%$ of units. At 150 to 350-ms time lags, LC micro-stimulation caused a combined facilitation followed by suppression on the evoked response. For lags of 400-ms and higher, the effect was pure facilitation. Additionally, response latency was significantly decreased at 250 ms time lag. In summary, we found that LC modulation affects the cortical processing of sensory inputs in a complex manner which critically depends on the time lag between LC activation and sensory input. These results have clear implications for temporal integration of sensory input and its noradrenergic modulation in a behavioral setting.
\end{abstract}




\section{Introduction}

Locus Coeruleus-norepinephrine (LC-NE) system has widespread projections throughout the brain and affects neuronal activity, network dynamics, and task-relevant perceptual behavior (Berridge \& Waterhouse, 2003; Sara, 2009; Sara \& Bouret, 2012; Waterhouse \& Navarra, 2019). Previous studies have revealed a prominent role of LC-NE system in controlling behavioral and brain states (Aston-Jones \& Bloom, 1981a; Berridge \& Waterhouse, 2003; Aston-Jones \& Cohen, 2005; Carter et al., 2010; Fazlali et al., 2016; Hayat et al., 2020) as well as modulation of sensory processing (Waterhouse et al., 1998; Berridge \& Waterhouse, 2003; Lecas, 2004; Manunta \& Edeline, 2004a; Devilbiss \& Waterhouse, 2004, 2011; Edeline et al., 2011; Vazey et al., 2018; Rodenkirch et al., 2019). Inhibition of both spontaneous and sensory-evoked cortical activity is well documented in classic experiments that used either LC electrical micro-stimulation or local application of norepinephrine (NE) (Foote et al., 1975; Phillis \& Kostopoulos, 1977). However, later studies reported more diverse effects on sensory response following LC electrical micro-stimulation or local NE application in sensory cortex and thalamus (Waterhouse et al., 1998; Berridge \& Waterhouse, 2003; Lecas, 2004; Manunta \& Edeline, 2004a; Devilbiss \& Waterhouse, 2004, 2011; Edeline et al., 2011; Waterhouse \& Navarra, 2019). The facilitation, suppression and the mixed effects of LC micro-stimulation on neuronal activity were found both in anesthetized (Lecas, 2004; Manunta \& Edeline, 2004b; Bouret \& Sara, 2004) and awake rats (Devilbiss \& Waterhouse, 2004, 2011; Devilbiss et al., 2006). In particular "signal-to-noise ratio" enhancement has been considered as a predominant effect of LC-NE system on the sensory response (Foote et al., 1975; Jiang et al., 1996; Waterhouse et al., 1998; Berridge \& Waterhouse, 2003; Hirata et al., 2006; Edeline, 2012). For example, LC activation was found to decrease spontaneous activity and increase the evoked response of neurons in the main olfactory bulb (Jiang et al., 1996). LC micro-stimulation enhanced the sensory-evoked responses in somatosensory cortical neurons without a significant effect on the spontaneous firing rate (Waterhouse et al., 1998; Bouret \& Sara, 2002). The maximum enhancement on the evoked somatosensory response was observed around 200-300 ms and diminished beyond 400-500 ms (Waterhouse et al., 1998).

Our aim here was to better characterize the temporal profile of LC stimulation on neuronal responses in the primary sensory cortex and the consequent effects on sensory processing. 
To this end, we recorded spiking activity in the rat barrel cortex and examined the LC-NE modulatory effects at systematic temporal intervals between LC and sensory (whisker) stimulation.

\section{Material and Methods}

\section{Surgery and electrophysiological recording}

Thirty-two adult male Wistar rats, weighing 270-390 g were used. All experiments were approved by the animal care and experimentation committee of the Institute for Research in Fundamental Science (IPM). Anesthesia was induced by intraperitoneal administration of urethane $(1.5 \mathrm{~g} / \mathrm{Kg})$, monitored by the hind paw and corneal reflexes, and maintained with supplemental doses of urethane $(0.1 \mathrm{~g} / \mathrm{Kg})$, if necessary. Two craniotomies were performed on the right hemisphere to provide access to $\mathrm{BC}(5 \times 5 \mathrm{~mm}$; centered at $2.6 \mathrm{~mm}$ posterior and $5 \mathrm{~mm}$ lateral to Bregma) and LC ( $4 \times 4 \mathrm{~mm}$; centered at $10.8 \mathrm{~mm}$ posterior to Bregma and $1.4 \mathrm{~mm}$ lateral to the midline). To facilitate access to LC, the rat's head was tilted down by about 14 degrees (Bouret \& Sara, 2002).

We recorded neuronal activity in BC while electrically stimulating ipsilateral LC (Figure $1 \mathrm{~A}$ ). $B C$ neuronal activity was acquired with single tungsten microelectrodes (1-2 $M \Omega$, FHC Inc., USA). The unit's principal whisker was determined by manual stimulation of individual whiskers. Layer 4 of the barrel cortex was targeted based on the depth of penetration from the surface of the exposed dura. Data were collected at a sampling rate of $30 \mathrm{kHz}$ and filtered on-line by applying a band-pass filter (300-6000 Hz). Spikes were sorted off-line using principal component analysis implemented in MATLAB (Math Works). In total, 49 multi-units were extracted from $\mathrm{BC}$ recordings for further analysis.

\section{Whisker stimulation}

Whisker stimuli (single cycle $80 \mathrm{~Hz}$ sinusoidal waveforms with various amplitudes) were delivered to the principal contra-lateral whisker using a piezoelectric device (NikTek, Tehran, Iran). Stimuli were generated in MATLAB and presented through the analog output of the sound card at a sampling rate of $96 \mathrm{kHz}$. The principal whisker was placed in a cannula connected to the piezo with a $2 \mathrm{~mm}$ distance from the tip of the cannula to the base of the whisker. To ensure precise whisker stimulation, we used an infrared optic sensor to calibrate the movement of the piezo. We measured the piezo movement to confirm that it 
accurately followed the voltage command with minimal resonation. For the range of stimulus intensities applied here $(6-60 \mu \mathrm{m})$, the post-deflection resonance was negligible ( $<6 \%$ of maximum amplitude) and was not detectable beyond $70-80 \mathrm{~ms}$. To select a nearthreshold range of stimuli, at the beginning of each recording session, we applied 10 levels of deflection from a relatively wide range of amplitudes $(0-60 \mu \mathrm{m}$ with $6 \mu \mathrm{m}$ steps, 50 repetitions). A Nuka-Rushton function was fitted to the average spike count to characterize the neuronal response function. Threshold $(T)$ was defined as the inflection point of this function - i.e. the stimulus amplitude that produced half of the maximum response (Fazlali et al., 2016).

In a subset of experiments $(n=33)$, the threshold stimulus was applied to the whisker at 10 time lags after LC micro-stimulation (from $50-500$ ms with 50 ms increment steps). In other experiments $(n=16)$ a range of near-threshold stimuli $(0,1 / 3 T, 2 / 3 T, T, 4 / 3 T, 5 / 3 T$, and $2 T)$ were applied to the principal whisker at 5 different time lags from LC micro-stimulation. In each session, deflections were applied in five blocks of 20 pseudo-randomized trials. Across five blocks each test stimulus was presented 100 times with 700-1000 ms random inter-trial interval. For each stimulus-amplitude, control (no micro-stimulation) trials were also intermixed with the main trials. In these trials, LC micro-stimulation preceded the whisker deflection by a minimum of $50 \mathrm{~ms}$. Interleaved with these trials, there were 100 trials with LC micro-stimulation alone (no whisker deflection). These trials allowed accurate estimation of the baseline activity at each of the time lags. This procedure was used to control for the tonic increase of NE in the cortex.

\section{LC micro-stimulation}

LC was targeted by single tungsten microelectrodes (<0.7 M 2 , FHC Inc., USA) from 5.6-5.9 $\mathrm{mm}$ below the dura. We used a monopolar stimulating electrode to minimize the damage to LC and its projections (Marzo et al., 2014). To confirm the LC location, we applied the following electrophysiological criteria: LC neurons normally have broad extracellular spike waveforms (>0.6 ms), firing rates of $0.1-6 \mathrm{~Hz}$, and respond to paw pinch with a typical excitation-inhibition pattern (Cedarbaum \& Aghajanian, 1978). At the end of the experiment, we verified the LC micro-stimulation site by histology. LC micro-stimulation consisted of a train of 3 bipolar pulses ( 0.5 ms pulse duration, $100 \mathrm{~Hz}, 30,50$ or $100 \mu \mathrm{A})$. A linear stimulus isolator (A395D, World Precision Instruments, Sarasota, FL, USA) was used to 
pass current through the electrodes. Due to the technical limitation, we were not able to simultaneously stimulate and record activity of LC. However, based on previous research currents within 3-300 $\mu \mathrm{A}$ range are expected to activate neurons within a maximal radius of $150 \mu \mathrm{m}$ (Stoney et al., 1968; John S. Yeomans, 1990; Snow et al., 1999). The precise timing and pattern of micro-stimulation was controlled by MATLAB and fed to the stimulator through analog output of an NI card (National Instruments, model: BNC 2090, Austin, TX, USA).

\section{Data analysis}

The sequences of spikes corresponding to trials of the same stimulus were separated and aligned with respect to stimulus onset to generate raster plots. The occurrence of spiking over time was evaluated by counting the average number of spikes within each time bin. A bin size of 10-20 ms was used to generate peristimulus time histograms (PSTHs). To quantify the effect of LC micro-stimulation on the evoked response, the spikes were counted within 1-75 ms and 125-250 ms after deflection (whisker stimulation) for early and late responses, respectively.

For spike time analysis, response delay was determined as the first time bin that exceeded background activity by 3 standard deviations (Figure 7).

\section{Histology}

At the end of the experiment, an electrical lesion was made by passing a DC current at $9 \mathrm{~V}$ through the LC electrode tip for $10 \mathrm{~s}$. Animals were sacrificed by transcranial perfusion with $\sim 300 \mathrm{ml}$ saline $(0.9 \%)$ followed by $\sim 300 \mathrm{ml}$ phosphate-buffered formalin $(10 \%, \mathrm{pH}=7.4)$. The brain was removed and kept in formalin (for a minimum of one week). Pontine coronal sections $(10 \mu \mathrm{m})$ were Nissl stained and lesions were detected by light microscopy. LC location was compared with the lesion site using the rat brain atlas (Paxinos and Watson, 2007). For all histological examinations, the micro-stimulation site was anatomically verified to be within LC confirming the reliability of our electrophysiological criteria.

\section{Results}

We electrically stimulated Locus Coeruleus (LC) while recording neuronal activity in the barrel cortex $(B C)$ of urethane anesthetized rats (Figure $1 A)$. LC recording was confirmed based on the characteristic spike waveform, the typical response profile to noxious 
stimulation, and histology (Figure $1 \mathrm{~B}$ and $\mathrm{C}$ ). $\mathrm{BC}$ recording was confirmed based on the neuronal response to brief deflections (single-cycle sinusoidal vibration, 12.5 ms duration) applied to the neuron's principal whisker. To identify the unit's threshold, at the beginning of each recording session, we applied 10 levels of deflection amplitudes (0-60 $\mu \mathrm{m}, 50$ repetitions) and fitted a Naka-Rushton function to the average spike count to characterize the neuronal response function. In the main experiment, we presented a range of deflections centered at the unit's threshold, T (see Materials and Methods). Figure 1B shows a typical multi-unit activity in three types of trials; control whisker deflection (brown), control LC micro-stimulation (gray), and combined LC stimulation and whisker deflection (blue).

\section{Modulation of spontaneous activity}

LC micro-stimulation exhibited a biphasic effect on BC spontaneous activity: a period of suppression ( $100 \mathrm{~ms}$ ) followed by a period of excitation ( $200 \mathrm{~ms})$. We applied LC microstimulation at three current levels; $30 \mu \mathrm{A}$ (7 sessions), $50 \mu \mathrm{A}$ (12 sessions), and $100 \mu \mathrm{A}$ (21 sessions). At $100 \mu \mathrm{A}$ current level, micro-stimulation produced a significant reduction of the average spontaneous spiking activity at 1-104 ms followed by an increase in firing rate at 117-325 ms (Figure 2A, top). Across units, maximum inhibition and excitation occurred at 40 ms and 195 ms, respectively. Beyond 325 ms, the spontaneous activity rarely deviated from its pre-stimulation average (Figure $2 \mathrm{~A}$, top). The modulation of spontaneous activity was smaller in sessions with $50 \mu \mathrm{A}$ current level (Figure 2A, middle) and even more diminished at $30 \mu \mathrm{A}$ current level (Figure 2A, bottom). On average, in sessions with $50-\mu \mathrm{A}$ current, inhibition occurred at 13-108 ms and excitation occurred at 122-309 ms. In $30 \mu \mathrm{A}$ current sessions, we observed a short period of excitation at 112-143 ms (on average) with no significant inhibition.

We further quantified the magnitude of suppression and excitation for individual units (50$\mu \mathrm{A}$ and $100-\mu \mathrm{A}$ current levels, $\mathrm{n}=33$ ). Based on the profile of modulation at the population level (Figure 2A), we chose two windows of 1-75 and 125-250 ms after LC micro-stimulation to respectively characterize the early and late phases of response. The suppressionexcitation profile was highly consistent across BC units: $82 \%$ of BC units showed early suppression and $91 \%$ of BC units showed late excitation (Figure 2B). Overall, the suppression was statistically significant for $70 \%$ (23/33) of units and the excitation was statistically 
significant for $76 \%(25 / 33$ ) of units (permutation test, $\mathrm{P}<0.05)$. At $30 \mu \mathrm{A}$ current level, the suppression and excitation modulation was not as consistent as that observed for the two higher current intensities; although the average firing rate profile did not show a prominent modulation (Figure 2A), 43\% (3/7) and 14\% (1/7) of units showed significant suppression and excitation (permutation test, $\mathrm{P}<0.05$ ) (Figure $2 \mathrm{~B}$ and $\mathrm{C}$ ). One multi-unit showed significant suppression at the second window (125-250 ms) in the opposite direction to other units (Figure $2 \mathrm{C}$ ).

There was no significant correlation between the amount of suppression and the baseline firing rate, indicating that the lack of suppression in some sessions was unlikely to be due to low firing rates ( $r h o=0.33, P=0.063, n=33$ ).

\section{Modulation of evoked activity}

How does LC micro-stimulation modulate the response profile of $B C$ neurons to sensory stimulation? To address this question, we stimulated LC in 10 time lags before the application of a single whisker deflection (50 ms to $500 \mathrm{~ms}, 50-\mathrm{ms}$ increments). LC microstimulation prominently decreased the early response in the barrel cortex at $50 \mathrm{~ms}$ time lag (Figure 3A and B). Overall, 77\% (30/39) of units showed a decrease in evoked firing rate (Figure 3B), and this decrease was statistically significant for $60 \%$ (100 $\mu \mathrm{A}$ stimulation), $25 \%$ (50 $\mu \mathrm{A}$ stimulation), and 14\% (30 $\mu \mathrm{A}$ stimulation) of the recorded units (permutation test, $\mathrm{P}$ $<0.05)$. In contrast to early response, the late activity increased in the majority of units, $74 \%$ (29/39); $16 / 20$ at $100 \mu \mathrm{A}$ stimulation, $9 / 12$ at $50 \mu \mathrm{A}$ stimulation, and $4 / 7$ at $30 \mu \mathrm{A}$ stimulation (Figure $3 C$ ). The enhanced late response was statistically significant for $50 \%$ of units at $50 \mu \mathrm{A}(6 / 12)$, and $100 \mu \mathrm{A}(10 / 20)$ (permutation test, $\mathrm{P}<0.05)$.

The micro-stimulation effect was highly dependent on the time lag: beyond 50 ms time lag, the early response prominently increased (see for example the $150 \mathrm{~ms}$ time lag in Figure 4A and B). Overall, $85 \%(33 / 39)$ of units showed an increase in firing rate (Figure 4B), and this increase was statistically significant for 45\% (100 $\mu \mathrm{A}$ stimulation), and $42 \%$ (50 $\mu \mathrm{A}$ stimulation) of the recorded units (permutation test, $\mathrm{P}<0.05$ ). Figure $4 \mathrm{C}$ summarizes the LC evoked modulation at all lags across all units $(n=33)$ for $100 \mu \mathrm{A}$ and $50 \mu \mathrm{A}$ currents.

The modulation of the evoked $\mathrm{BC}$ response observed in the previous section could simply reflect the modulation of the spontaneous activity as quantified earlier. To examine this 
possibility, we subtracted for each time lag the peri-stimulus time histogram (PSTH) in the absence of LC micro-stimulation from the one with LC micro-stimulation (Figure 5A). This residual PSTH reflects the changes in firing rate that are produced as a result of LC microstimulation (referred to as the Modulation Index in Figure 5C, D). We then overlaid this residual PSTH (black curves) on the spontaneous firing rate (baseline subtracted, gray curves) for each specific time lag (Figure 5A). At 50 ms time lag, the suppression of earlyevoked response ( $75 \mathrm{~ms}$ from deflection onset) was on average higher than the corresponding suppression in spontaneous activity (Figure 5A). The late response (125-250 $\mathrm{ms}$ ) at $50 \mathrm{~ms}$ time lag tended to increase above the changes that were expected based on the spontaneous profile (Figure 5B). At $100 \mathrm{~ms}$ time lag, the early suppression was shorter and less pronounced but the late facilitation was comparable with that of $50 \mathrm{~ms}$ time lag. At $150 \mathrm{~ms}$ time lag, the early-evoked response was substantially facilitated and the late-evoked response was not significantly modulated compared to the spontaneous activity (Figure 5A). Beyond 150 ms time lag, we observed little modulation of activity with the exception of a slight facilitation in the initial phase of the response (see the $500 \mathrm{~ms}$ time lag in Figure 5B). Across all time lags, the activity beyond $700 \mathrm{~ms}$ from the deflection onset was similar to the spontaneous activity in the control condition. The modulations at $50 \mu \mathrm{A}$ current were significantly lower compared to $100 \mu \mathrm{A}$ (for example for $50-\mathrm{ms}$ lag) but were qualitatively similar. At $30 \mu \mathrm{A}$, although some units were affected by LC micro-stimulation, on average the modulation pattern was absent.

Finally, we asked whether the modulation of spontaneous activity could explain the modulation of evoked response across recording sessions. Figure 5B plots the spontaneous modulation index against the early-evoked modulation index (1-75 ms from the deflection onset). At 50 ms time lag across the 3 current levels, $77 \%$ of the sessions showed higher evoked modulation than spontaneous ( $n=30 / 39)$. Notably, the sessions with little modulation of spontaneous activity had comparable (permutation test, $\mathrm{P}=0.27$ ) baseline firing rate with those which had stronger spontaneous modulation (red boxes in figure 5C), indicating that the difference between evoked and spontaneous modulation is not due to a floor effect when baseline activity might be too low to exhibit suppression of firing rate). At $150 \mathrm{~ms}$ lag, the evoked modulation was higher than spontaneous modulation in $64 \%$ of the sessions ( $n=25 / 39$ ). For 6 units outlined with a red box in Figure 5C, spontaneous activity 
was not significantly modulated in 5 units (permutation test), while the evoked response was substantially enhanced (Figure 5C, 150 early). In contrast, in 250 ms lag, 64\% of the units showed higher spontaneous modulation indicating a relative suppression of the evoked response. Unlike the early response, for the majority of units, the late activity did not show a significant modulation above the spontaneous profile: at 50-ms time lag, for the $125-250$ ms post deflection onset, modulation of evoked activity paralleled that of spontaneous activity (note that the most of the most units are dispersed along the diagonal line in Figure 5C). This is consistent with a linear summation of the LC-evoked spikes and deflection-evoked spikes and hence no net modulation of the evoked response. Still, for a few units, LC stimulation has increased (or decreased, Figure 5D) the net value of the late evoked response.

\section{Modulation of response latency}

The average profile of modulation in Figure 5 suggests that LC micro-stimulation can affect the temporal structure of the response. For example, in 50 and 100 ms time lags, we observed a suppression followed by facilitation in the modulation profile (Figure 5B). We, therefore, asked if LC stimulation affected the neuronal response latencies to sensory stimulation. Figure $6 \mathrm{~A}$ quantifies the modulation of response latency across all time lags (see Materials and methods). Although the average response latency appeared to increase at $100 \mathrm{~ms}$ lag, this change was not statistically significant $(P=0.055$; control condition, 12.8 \pm 0.6 and LC micro-stimulation, $13.9 \pm 0.6$; Figure $6 \mathrm{~B})$. However, the coupling of deflection with LC micro-stimulation at $250 \mathrm{~ms}$ time lag significantly decreased the response latency from $12.8 \pm 0.6$ to $11.8 \pm 0.6(P<0.001$, permutation test, Figure $6 C)$.

\section{Modulation of the stimulus-response function}

How does LC micro-stimulation affect the stimulus-response function and the sensitivity of neurons in detecting sensory stimuli? To address this question, in a subset of experiments we applied a range of near-threshold stimuli; $1 / 3 \mathrm{~T}, 2 / 3 \mathrm{~T}, \mathrm{~T}, 4 / 3 \mathrm{~T}, 5 / 3 \mathrm{~T}$, and $2 \mathrm{~T}$ and obtained a full stimulus-response function in the presence and absence of LC activation (Figure 7). LC micro-stimulation at $50 \mathrm{~ms}$ lag decreased the neuronal response at all stimulus intensities (Figure 7A, top). On the other hand, at $150 \mathrm{~ms}$ lag, LC micro-stimulation increased the response at all stimulus intensities (Figure 7A, middle). This enhancement of response 
diminished at $250 \mathrm{~ms}$ lag (Figure 7A, bottom). The overall upward shift in the response function resulted in a reduced response range (Figure 7B).

\section{Discussion}

Here, we studied the temporal profile of LC modulation of sensory processing by measuring how phasic LC micro-stimulation affected the spontaneous and stimulus-evoked activity in the rat barrel cortex across a range of time lags (50-500 ms). LC micro-stimulation exhibited a biphasic effect on BC spontaneous activity: a period of suppression followed by a period of excitation consistent with earlier experiments (Phillis \& Kostopoulos, 1977). The effect of LC micro-stimulation on the evoked response to the near-threshold stimulus was highly timedependent: at 50-ms lag, the early-evoked response decreased in the majority of units. This was in contrast to $150-\mathrm{ms}$ lag, where the majority of units exhibited response facilitation. At 150 to 350-ms time lags, LC micro-stimulation caused a combined facilitation followed by suppression. At higher time lags $(\geq 400)$, the effect was mainly facilitation. Response latency decreased at $250 \mathrm{~ms}$ time lag.

\section{LC micro-stimulation and sensory processing}

The effect of LC micro-stimulation on sensory responses is often quantified in time lags between 250 to 350 ms (Bouret \& Sara, 2002; Lecas, 2004; Edeline et al., 2011). LC responds to sensory stimuli with a short latency in awake animals (Foote et al., 1980; Aston-Jones \& Bloom, 1981b). A previous study compared the modulation of sensory response in the somatosensory cortex at time intervals of $100-500 \mathrm{~ms}$ and found mainly potentiation with a peak at 200 ms (Waterhouse et al., 1998). However, micro-stimulation parameters in such studies are often adjusted to be sub-threshold for modulation of spontaneous activity (Waterhouse et al., 1998; Lecas et al., 2001; Lecas, 2004). In our study, different current levels were used to compare the relation between modulation of spontaneous and evoked activities. Our data suggest that although the modulation of sensory response by LC microstimulation lasts for a long period (at least $500 \mathrm{~ms}$ ), the peak of effects can emerge within $100 \mathrm{~ms}$ as a strong suppression of both spontaneous and evoked activity, followed by facilitation at 150 ms time lag. We found various degrees of correlation between spontaneous and evoked activity; in some sessions, evoked activity was modulated independently of modulation in spontaneous activity (Figure 5B). We observed less 
modulation in evoked activity when the current was adjusted to have a minimal effect on spontaneous activity (30 $\mu \mathrm{A}$, Figure $5 \mathrm{~B})$.

\section{Effectiveness and specificity of $L C$ activation}

The level of activation of brain tissue by electrical stimulation depends on multiple parameters such as current pulse amplitude, polarity, duration and frequency (Mclntyre \& Grill, 2000; Tehovnik et al., 2006). Since myelinated axon fibers have higher excitability (Nowak \& Bullier, 1998; Tehovnik et al., 2006), the spread of current in pons may directly activate the surrounding fibers. This can affect other cortical areas with a chain of transsynaptic activations/inhibitions resulting in the observed effects. Consistent with this possibility, we observed bilateral facial movements with $100 \mu \mathrm{A}$ current in several recording experiments (data not included). With lower currents (50 and $30 \mu \mathrm{A}$ ), no facial movement was observed. A study reported that this level of stimulation (30 and $50 \mu \mathrm{A}, 0.4 \mathrm{~ms}$ duration) can produce bilateral modulation of LC activity (Marzo et al., 2014). Given that there is no direct connection between the two sides of LC (Aston-Jones et al., 1991), the possibility of direct activation of contralateral LC that is located more than $2 \mathrm{~mm}$ away from the stimulating electrode (Marzo et al., 2014) cannot be ruled out. Likewise, in our study the direct or indirect activation of other structures, including pontine reticular formation and basal forebrain (Dringenberg \& Vanderwolf, 1997) cannot be ruled out. Future optogenetic experiments can directly address this concern.

We presented a range of whisker stimuli to quantify the effect of LC micro-stimulation on stimulus-response function. At least one study has shown that phasic LC stimulation, $300 \mathrm{~ms}$ before whisker deflection, causes a leftward shift in stimulus-response function and greatly facilitates response to weaker stimuli in the thalamus (Berridge \& Waterhouse, 2003).

In our data, the suppression at 50-ms lag and the facilitation at 150-250 ms was consistently observed for near-threshold stimuli. On the other hand, the response to the highest stimulus intensity $(2 \mathrm{~T})$ remained unchanged with $\mathrm{LC}$ micro-stimulation (Figure $7 \mathrm{~A}$ ). We found that strong LC micro-stimulation is likely to affect near-threshold stimuli, decrease the range of responsiveness (Figure 7B), and disrupt the encoding. 


\section{LC activity and cortical state}

The role of LC-NE system in changing cortical state is implicated in several studies (Lee \& Dan, 2012; Marzo et al., 2014; Zagha \& McCormick, 2014; Fazlali et al., 2016; Hayat et al., 2020; Ranjbar-Slamloo \& Fazlali, 2020). The change in cortical state can be quantified using a low-to-high (LH) EEG power ratio (Fazlali et al. 2016). In this study, we did not observe any significant change in the LH ratio as an index of brain state. There is a correlation between LC tonic activity and two extreme cortical states; low LC firing rate with synchronized state and high LC firing rate with desynchronized state (Aston-Jones \& Bloom, 1981a; Fazlali et al., 2016). However, there is growing evidence showing that the cortical state is more diverse than just two extremely activated and deactivated states. The interplay between various neuromodulatory systems and cortical state is complex (Harris \& Thiele, 2011; Zagha \& McCormick, 2014; McGinley et al., 2015a, 2015b; Sabri \& Arabzadeh, 2018). For instance, McGinley et al showed that the effect of cortical state on sensory response is $U$ shape; low arousal, moderate arousal and high arousal. In moderate arousal where the index of the cortical state is in a middle range, sensory processing is optimum (McGinley et al., 2015a). It is however not clear how phasic LC micro-stimulation changes the network state.

\section{LC topography, projections and their effect on BC spontaneous activity}

The effect of LC micro-stimulation with 100 and $50 \mu \mathrm{A}$ on spontaneous activity showed a biphasic profile; early inhibition and late excitation (Figure 2A). LC neurons projecting to cortical and subcortical areas are not homogenously distributed across this nucleus (Sara \& Bouret, 2012; Schwarz \& Luo, 2015; Breton-Provencher \& Sur, 2019). Cortical projecting neurons are located at the body, while thalamic projecting neurons are located in the posterior tail of LC (Loughlin et al., 1986; Schwarz \& Luo, 2015). Through dense projections to the thalamic reticular nucleus (TRN), LC can gate information flow in the whisker pathway (Rodenkirch et al., 2019). High variability that we observed across sessions might be due to session-to-session variability in targeting different parts of LC (Totah et al., 2018; BretonProvencher \& Sur, 2019). Moreover, the biphasic nature of the cortical modulation caused by larger currents might be due to the activation of separate LC-NE projecting pathways (Logothetis et al., 2010). 


\section{LC activity and behavior}

The central nervous system's ability to efficiently extract relevant information from the sensory environment is essential for survival. The whisker system is one of the main channels through which rodents collect information from their environment (Diamond \& Arabzadeh, 2013). Behavioral studies have revealed a tight correlation between neuronal activity in the barrel cortex and whisker mediated behavior (von Heimendahl et al., 2007; O'Connor et al., 2010, 2013). These behavioral studies typically use well-trained animals with reliable levels of performance. The degree of synchronization in cortical cells is shown to depend on the level of training: naïve animals elicit a more synchronized cortical state compared to trained animals (Sachidhanandam et al., 2013). The LC-NE modulation of the cortical state may play a role in improved performance observed with training. Consistent with this idea, the activity of the LC-NE system is shown to predict the behavioral performance of primates (Aston-Jones et al., 1999; Aston-Jones \& Cohen, 2005).

\section{Concluding remarks}

The majority of studies on LC-NE system and sensory properties suggest a role for this system in perceptual acuity (Sara, 2009; Waterhouse \& Navarra, 2019). Our data suggest that time lag of coupling between LC activation and sensory input can be a crucial parameter for the interpretation of LC-NE neuromodulatory effects with the microstimulation approach. Not only LC, the parameters of micro-stimulations and time lag of modulatory effects may also apply to other neuromodulatory nuclei. Although there is growing studies that activated LC optogenetically (Carter et al., 2010; Breton-Provencher \& Sur, 2019; Hayat et al., 2020), future studies could selectively activate LC and record from sensory cortices to confirm the specificity of the observed modulations both in spontaneous and evoked activities. 


\section{Author Contributions}

ZF, YR-S, and EA designed research; ZF and YR-S performed experiments; ZF analyzed the data; ZF, YR-S, and EA interpreted results of experiments; ZF prepared figures; ZF drafted manuscript; ZF, YR-S, and EA edited and revised manuscript.

\section{Conflict of Interest Statement}

The authors declare that the research was conducted in the absence of any commercial or financial relationships that could be construed as a potential conflict of interest. 


\section{References}

Aston-Jones G \& Bloom FE (1981a). Activity of norepinephrine-containing locus coeruleus neurons in behaving rats anticipates fluctuations in the sleep-waking cycle. J Neurosci 1, 876-886.

Aston-Jones G \& Bloom FE (1981b). Norepinephrine-containing locus coeruleus neurons in behaving rats exhibit pronounced responses to non-noxious environmental stimuli. J Neurosci 1, 887900.

Aston-Jones G \& Cohen JD (2005). An integrative theory of locus coeruleus-norepinephrine function: adaptive gain and optimal performance. Annu Rev Neurosci 28, 403-450.

Aston-Jones G, Rajkowski J \& Cohen J (1999). Role of Locus Coeruleus in Attention and Behavioral Flexibility. Biol Psychiatry 46, 1309-1320.

Aston-Jones G, Shipley MT, Chouvet G, Ennis M, van Bockstaele E, Pieribone V, Shiekhattar R, Akaoka H, Drolet G, Astier B, Charléty P, Valentino RJ \& Williams JT (1991). Neurobiology of The Locus Coeruleus. Elsevier.

Berridge CW \& Waterhouse BD (2003). The locus coeruleus- noradrenergic system: modulation of behavioral state and state-dependent cognitive processes. Brain Res Rev 42, 33-84.

Bouret S \& Sara SJ (2002). Locus coeruleus activation modulates firing rate and temporal organization of odour-induced single-cell responses in rat piriform cortex. Eur J Neurosci 16, 2371-2382.

Bouret S \& Sara SJ (2004). Reward expectation, orientation of attention and locus coeruleus-medial frontal cortex interplay during learning. Eur J Neurosci 20, 791-802.

Breton-Provencher V \& Sur M (2019). Active control of arousal by a locus coeruleus GABAergic circuit. Nat Neurosci 22, 218-228.

Carter ME, Yizhar O, Chikahisa S, Nguyen H, Adamantidis A, Nishino S, Deisseroth K \& de Lecea L (2010). Tuning arousal with optogenetic modulation of locus coeruleus neurons. Nat Neurosci 13, 1526-1533.

Cedarbaum JM \& Aghajanian GK (1978). Activation of locus coeruleus neurons by peripheral stimuli: modulation by a collateral inhibitory mechanism. Life Sci $\mathbf{2 3}, \mathbf{1 3 8 3 - 1 3 9 2 .}$

Devilbiss DM, Page ME \& Waterhouse BD (2006). Locus ceruleus regulates sensory encoding by neurons and networks in waking animals. J Neurosci 26, 9860-9872.

Devilbiss DM \& Waterhouse BD (2004). The effects of tonic locus ceruleus output on sensory-evoked 
responses of ventral posterior medial thalamic and barrel field cortical neurons in the awake rat. J Neurosci 24, 10773-10785.

Devilbiss DM \& Waterhouse BD (2011). Phasic and tonic patterns of locus coeruleus output differentially modulate sensory network function in the awake rat. J Neurophysiol 105, 69-87.

Diamond ME \& Arabzadeh E (2013). Whisker sensory system - From receptor to decision. Prog Neurobiol 103, 28-40.

Dringenberg HC \& Vanderwolf CH (1997). Neocortical activation: modulation by multiple pathways acting on central cholinergic and serotonergic systems. Exp Brain Res 116, 160-174.

Edeline J-M (2012). Beyond traditional approaches to understanding the functional role of neuromodulators in sensory cortices. Front Behav Neurosci 6, 45.

Edeline J-M, Manunta Y \& Hennevin E (2011). Induction of selective plasticity in the frequency tuning of auditory cortex and auditory thalamus neurons by locus coeruleus stimulation. Hear Res 274, 75-84.

Fazlali Z, Ranjbar-Slamloo Y, Adibi M \& Arabzadeh E (2016). Correlation between Cortical State and Locus Coeruleus Activity: Implications for Sensory Coding in Rat Barrel Cortex. Front Neural Circuits; DOI: 10.3389/fncir.2016.00014.

Foote SL, Aston-Jones G \& Bloom FE (1980). Impulse activity of locus coeruleus neurons in awake rats and monkeys is a function of sensory stimulation and arousal. Proc Natl Acad Sci U S A 77, 3033-3037.

Foote SL, Freedman R \& Oliver AP (1975). Effects of putative neurotransmitters on neuronal activity in monkey auditory cortex. Brain Res 86, 229-242.

Harris KD \& Thiele A (2011). Cortical state and attention. Nat Rev Neurosci 12, 509-523.

Hayat H, Regev N, Matosevich N, Sales A, Paredes-Rodriguez E, Krom AJ, Bergman L, Li Y, Lavigne M, Kremer EJ, Yizhar O, Pickering AE \& Nir Y (2020). Locus coeruleus norepinephrine activity mediates sensory-evoked awakenings from sleep. Sci Adv; DOI: 10.1126/sciadv.aaz4232.

von Heimendahl M, Itskov PM, Arabzadeh E \& Diamond ME (2007). Neuronal activity in rat barrel cortex underlying texture discrimination. PLoS Biol 5, e305.

Hirata A, Aguilar J \& Castro-Alamancos M a (2006). Noradrenergic activation amplifies bottom-up and top-down signal-to-noise ratios in sensory thalamus. J Neurosci $\mathbf{2 6 , 4 4 2 6 - 4 4 3 6 .}$

Jiang M, Griff ER, Ennis M, Zimmer LA \& Shipley MT (1996). Activation of Locus Coeruleus Enhances 
the Responses of Olfactory Bulb Mitral Cells to Weak Olfactory Nerve Input. 16, 6319-6329.

John S. Yeomans (1990). Principles of Brain Stimulation.

Lecas J (2004). Locus coeruleus activation shortens synaptic drive while decreasing spike latency and jitter in sensorimotor cortex . Implications for neuronal integration. Eur J Neurosci 19, 25192530.

Lecas J, Neuromodulation D, Neurosciences I, Pierre U \& Bât B (2001). Noradrenergic modulation of tactile responses in rat cortex . Current source-density and unit analyses. 324, 33-44.

Lee S-H \& Dan Y (2012). Neuromodulation of brain states. Neuron 76, 209-222.

Logothetis NK, Augath M, Murayama Y, Rauch A, Sultan F, Goense J, Oeltermann A \& Merkle H (2010). The effects of electrical microstimulation on cortical signal propagation. Nat Neurosci $13,1283-1291$.

Loughlin SE, Foote SL \& Bloom FE (1986). Efferent projections of nucleus locus coeruleus:

Topographic organization of cells of origin demonstrated by three-dimensional reconstruction. Neuroscience 18, 291-306.

Manunta Y \& Edeline J-M (2004a). Noradrenergic induction of selective plasticity in the frequency tuning of auditory cortex neurons. J Neurophysiol 92, 1445-1463.

Manunta Y \& Edeline J (2004b). Noradrenergic Induction of Selective Plasticity in the Frequency Tuning of Auditory Cortex Neurons Noradrenergic Induction of Selective Plasticity in the Frequency Tuning of Auditory Cortex Neurons. J Neurophysiol 92, 1445-1463.

Marzo A, Totah NK, Neves RM, Logothetis NK \& Eschenko O (2014). Unilateral electrical stimulation of rat locus coeruleus elicits bilateral response of norepinephrine neurons and sustained activation of medial prefrontal cortex. J Neurophysiol 111, 2570-2588.

McGinley MJ, David SV \& McCormick DA (2015a). Cortical Membrane Potential Signature of Optimal States for Sensory Signal Detection. Neuron 87, 179-192.

McGinley MJ, Vinck M, Reimer J, Batista-Brito R, Zagha E, Cadwell CR, Tolias AS, Cardin JA \& McCormick DA (2015b). Waking state: rapid variations modulate neural and behavioral responses. Neuron $\mathbf{8 7}, 1143-1161$.

McIntyre CC \& Grill WM (2000). Selective microstimulation of central nervous system neurons. Ann Biomed Eng 28, 219-233.

Nowak LG \& Bullier J (1998). Axons, but not cell bodies, are activated by electrical stimulation in 
cortical gray matter. I. Exp Brain Res 118, 489-500.

O'Connor DH, Clack NG, Huber D, Komiyama T, Myers EW \& Svoboda K (2010). Vibrissa-based object localization in head-fixed mice. J Neurosci 30, 1947-1967.

O’Connor DH, Hires SA, Guo Z V, Li N, Yu J, Sun Q-Q, Huber D \& Svoboda K (2013). Neural coding during active somatosensation revealed using illusory touch. Nat Neurosci 16, 958-965.

Paxinos G \& Watson C (2007). The Rat Brain in Stereotaxic Coordinates. Academic, San Diego, USA.

Phillis JW \& Kostopoulos GK (1977). Activation of a noradrenergic pathway from the brain stem to rat cerebral cortex. Gen Pharmacol 8, 207-211.

Ranjbar-Slamloo Y \& Fazlali Z (2020). Dopamine and Noradrenaline in the Brain; Overlapping or Dissociate Functions? Front Mol Neurosci 12, 1-8.

Rodenkirch C, Liu Y, Schriver BJ \& Wang Q (2019). Locus coeruleus activation enhances thalamic feature selectivity via norepinephrine regulation of intrathalamic circuit dynamics. Nat Neurosci 22, 120-133.

Sabri MM \& Arabzadeh E (2018). Information processing across behavioral states: Modes of operation and population dynamics in rodent sensory cortex. Neuroscience 368, 214-228.

Sachidhanandam S, Sreenivasan V, Kyriakatos A, Kremer Y \& Petersen CCH (2013). Membrane potential correlates of sensory perception in mouse barrel cortex. Nat Neurosci 16, 1671-1677. Sara SJ (2009). The locus coeruleus and noradrenergic modulation of cognition. Nat Rev Neurosci 10, 211-223.

Sara SJ \& Bouret S (2012). Orienting and reorienting: the locus coeruleus mediates cognition through arousal. Neuron 76, 130-141.

Schwarz LA \& Luo L (2015). Organization of the Locus Coeruleus-Norepinephrine System. Curr Biol 25, R1051-R1056.

Snow PJ, Andre P \& Pompeiano O (1999). Effects of locus coeruleus stimulation on the responses of SI neurons of the rat to controlled natural and electrical stimulation of the skin. Arch Ital Biol $137,1-28$.

Stoney SDJ, Thompson WD \& Asanuma H (1968). Excitation of pyramidal tract cells by intracortical microstimulation: effective extent of stimulating current. J Neurophysiol 31, 659-669.

Tehovnik EJ, Tolias a S, Sultan F, Slocum WM \& Logothetis NK (2006). Direct and indirect activation of cortical neurons by electrical microstimulation. J Neurophysiol 96, 512-521. 
Totah NK, Neves RM, Panzeri S, Logothetis NK \& Eschenko O (2018). The Locus Coeruleus Is a Complex and Differentiated Neuromodulatory System. Neuron 99, 1055-1068.e6.

Vazey EM, Moorman DE \& Aston-Jones G (2018). Phasic locus coeruleus activity regulates cortical encoding of salience information. Proc Natl Acad Sci U S A 115, E9439-E9448.

Waterhouse BD, Moises HC \& Woodward DJ (1998). Phasic activation of the locus coeruleus enhances responses of primary sensory cortical neurons to peripheral receptive field stimulation. Brain Res 790, 33-44.

Waterhouse BD \& Navarra RL (2019). The locus coeruleus-norepinephrine system and sensory signal processing: A historical review and current perspectives. Brain Res 1709, 1-15.

Zagha E \& McCormick D a (2014). Neural control of brain state. Curr Opin Neurobiol 29C, 178-186. 


\section{Figure 1}

A

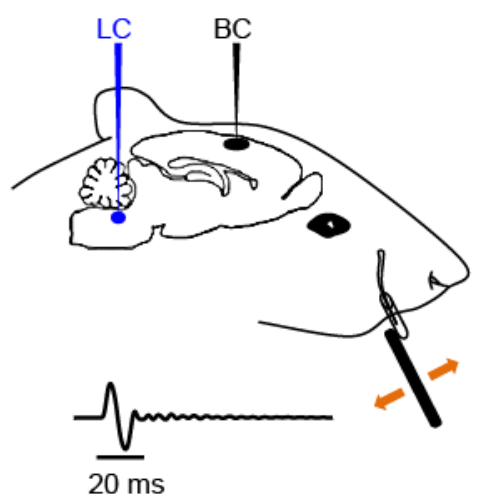

B

C
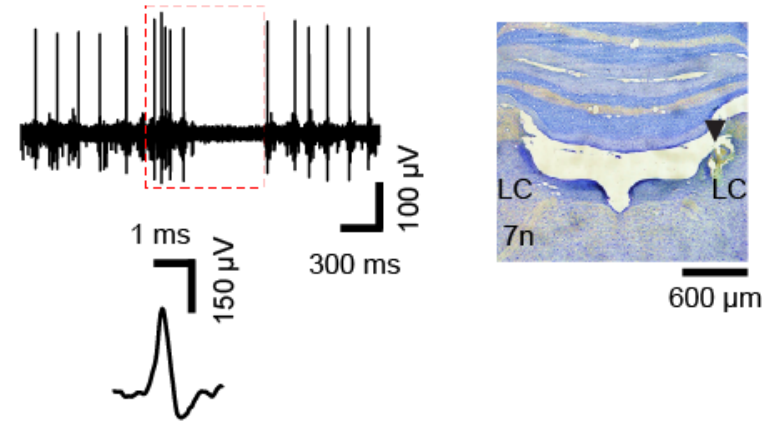

D
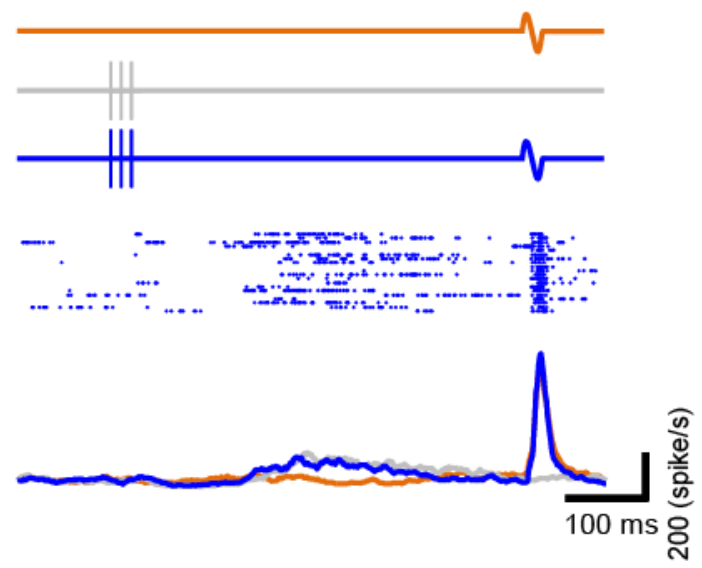

Figure 1. Schematic illustration of the experiment.

(A) Neuronal activity was recorded from Barrel Cortex (BC) while a brief mechanical vibration was applied to the contralateral whisker, at various intervals relative to micro-stimulation of 
Locus Coeruleus (LC). For sensory stimulation, a full-cycle sinusoidal deflection was applied to the contralateral whisker. $B$ and $C$ are from your previous paper. (D) There were three trial types: those with whisker deflections but no LC micro-stimulation (brown); those with LC microstimulation but no whisker deflections (gray); and those that contained both LC microstimulation and whisker deflections (blue). Raster plot shows the firing pattern of a typical multi-unit during LC micro-stimulation-deflection trials. Lower PSTHs show the firing rate of this typical multi-unit in three different trial types. This color convention will be used henceforth. 
Figure 2

A

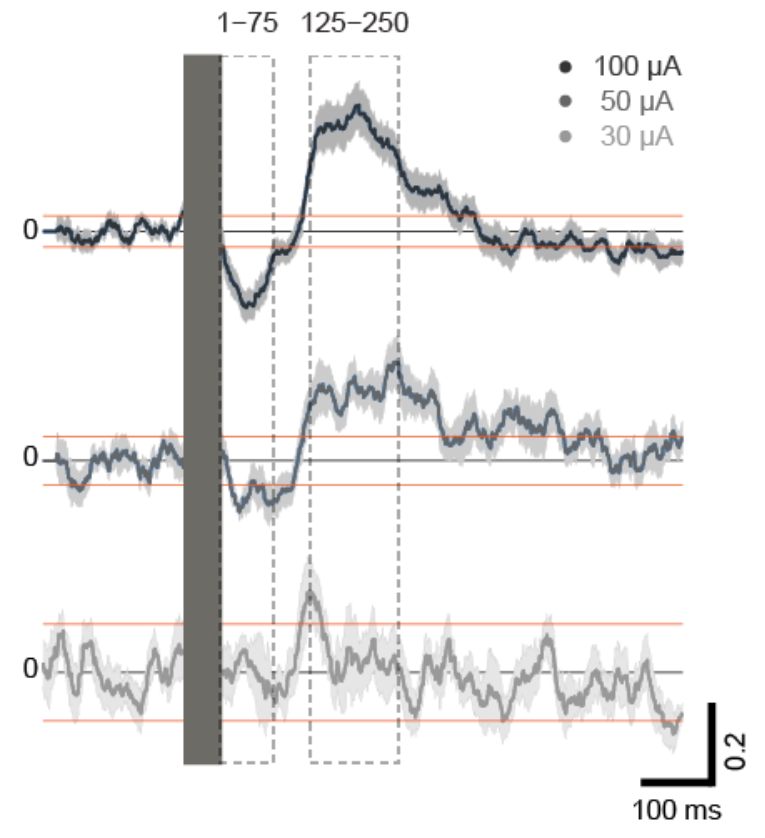

B
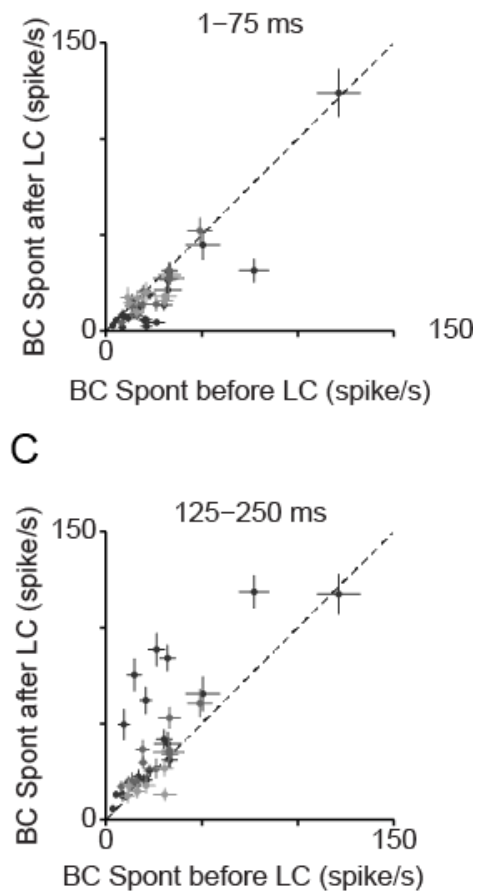

D

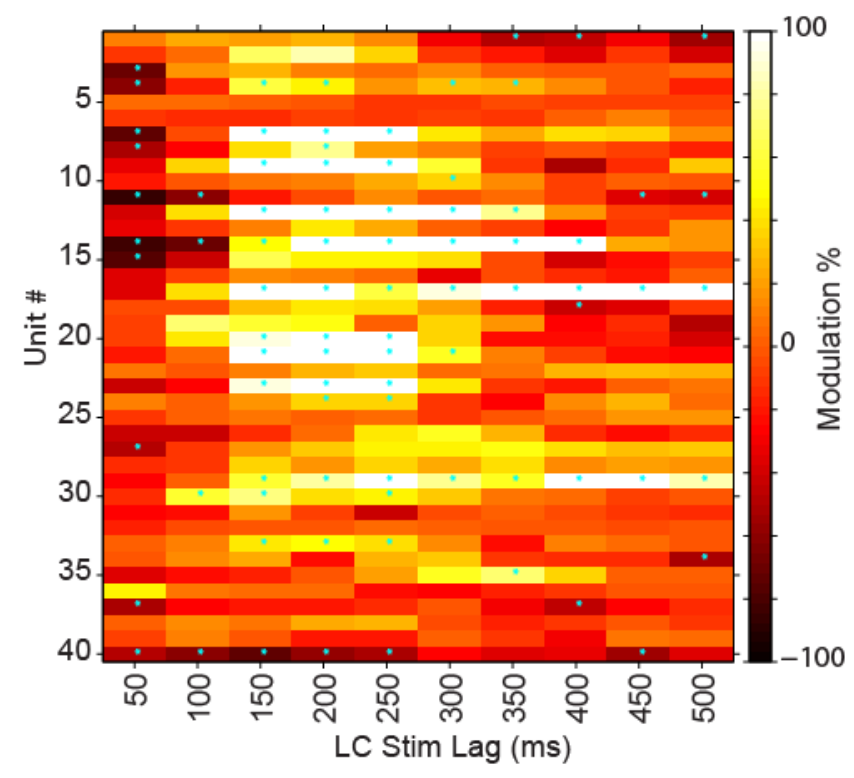


Figure 2. Effect of LC micro-stimulation on BC spontaneous activity.

(A) Average of BC spontaneous activity before and after LC micro-stimulation (solid box) across multi-units. Each row shows one level of current injection for LC micro-stimulation: $100 \mu \mathrm{A}$ (top), $50 \mu \mathrm{A}$ (middle) and $30 \mu \mathrm{A}$ (bottom). Shaded error bars show standard error of mean (SEM) across multi-units. (B) Every dot represents one multi-unit. Responses show suppression during the early window (1-75 ms post-stimulus onset as outlined in panel A). Error bars show standard error of mean (SEM) across trials for each unit in $\mathrm{x}$ and $\mathrm{y}$ axes. (C) Same as panel $\mathrm{B}$ but for the late excitation phases (125-250 ms, as outlined in panel A). (D) Spontaneous modulation of all units at 10 different time lags. 


\section{Figure 3}

A

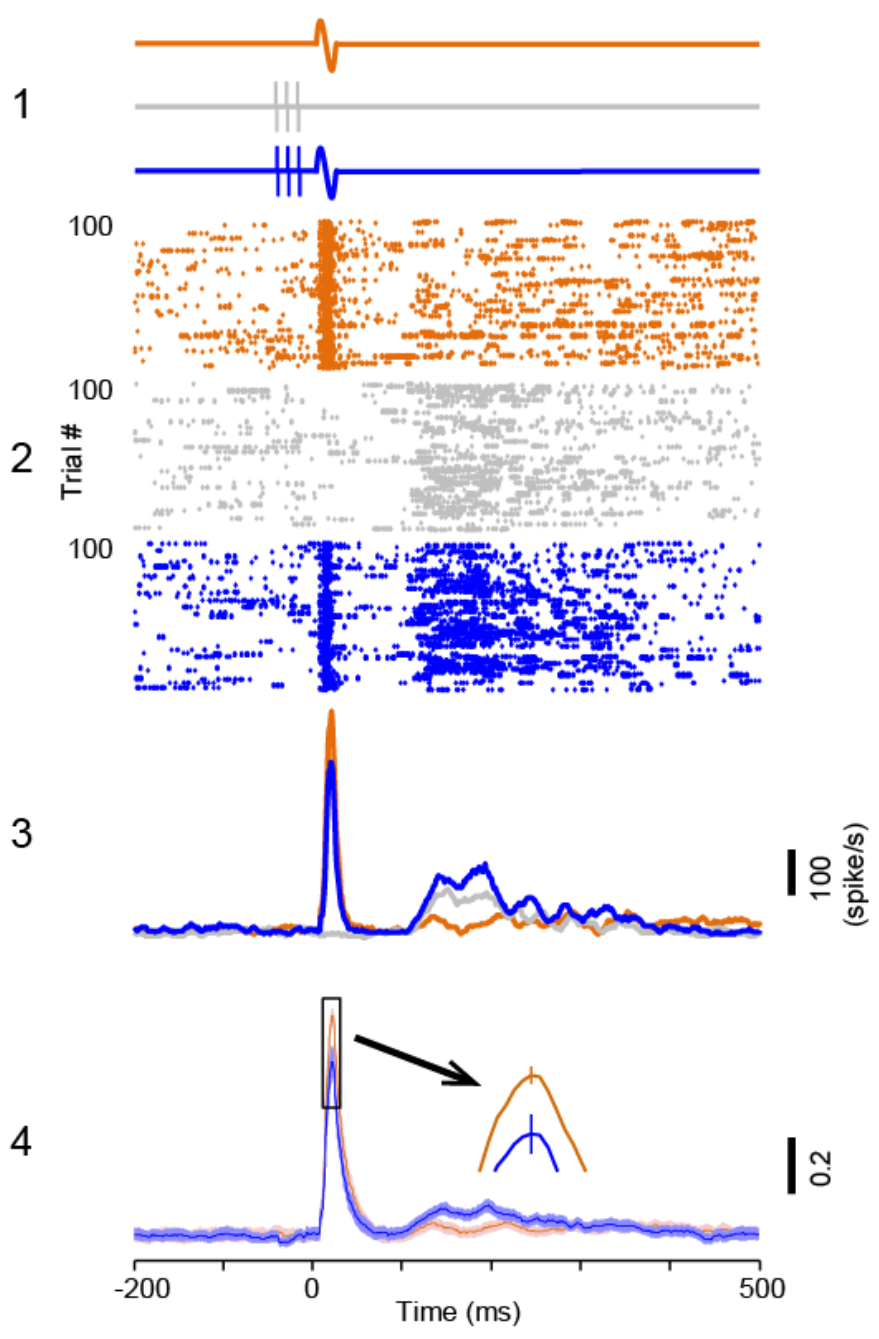

B

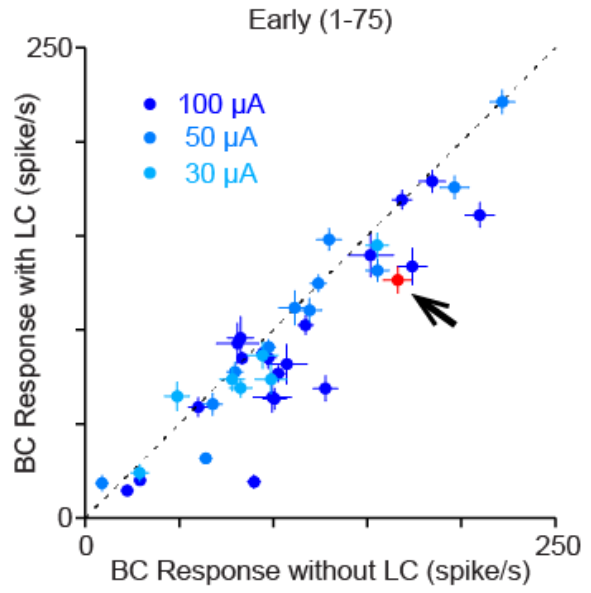

C

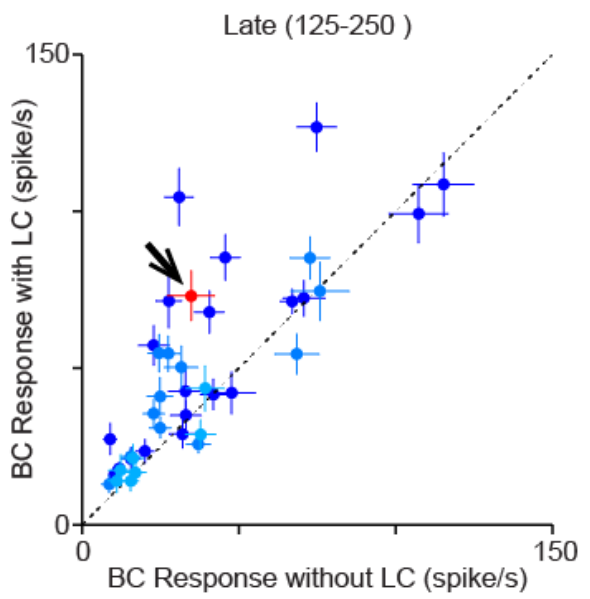

Figure 3. Suppression of BC evoked response by LC micro-stimulation.

(A1) LC was micro-stimulated 50-ms before whisker deflection. LC micro-stimulation current was $100 \mu \mathrm{A}$. (A2) Raster plot of a typical multi-unit in three types of trials: those with whisker deflections but no LC micro-stimulation (brown); those with LC micro-stimulation but no whisker deflections (gray); and those that contained both LC micro-stimulation and whisker deflections (blue). (A3) PSTHs of the typical unit in A2. (A4) Average of normalized PSTHs across all recorded multi-units. Shaded error bars show standard error of mean (SEM) across multiunits. (B) Scatter plot of the BC early response (1-75) during LC micro-stimulation versus no LC 
micro-stimulation condition. Each dot-color shows one unit-current level. Error bars show SEM across trials in each axis. A black arrow (red dot) shows a representative unit in panel A. (C) Same as B but for later phase of the evoked response (125-250 ms). 


\section{Figure 4}

A

1

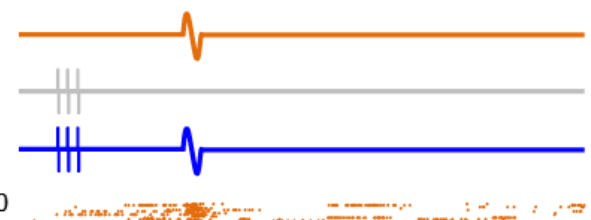

100

100

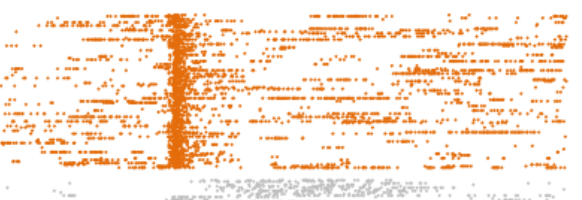

2

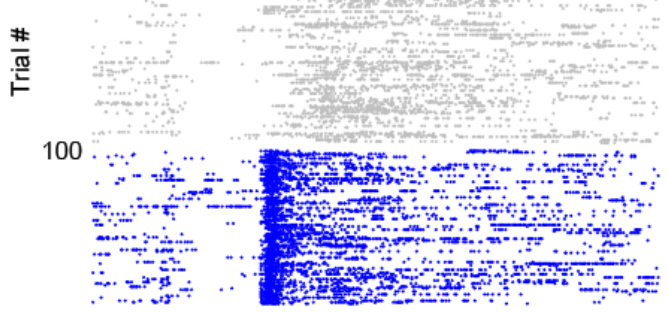

3

4

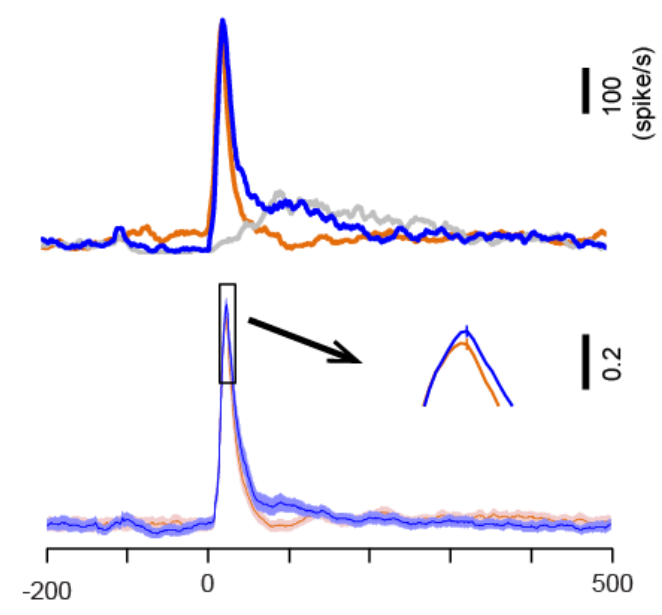

B

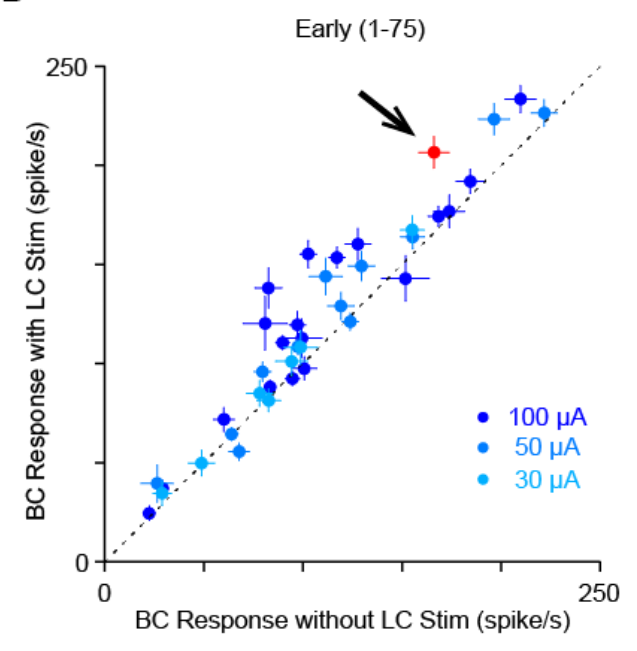

C

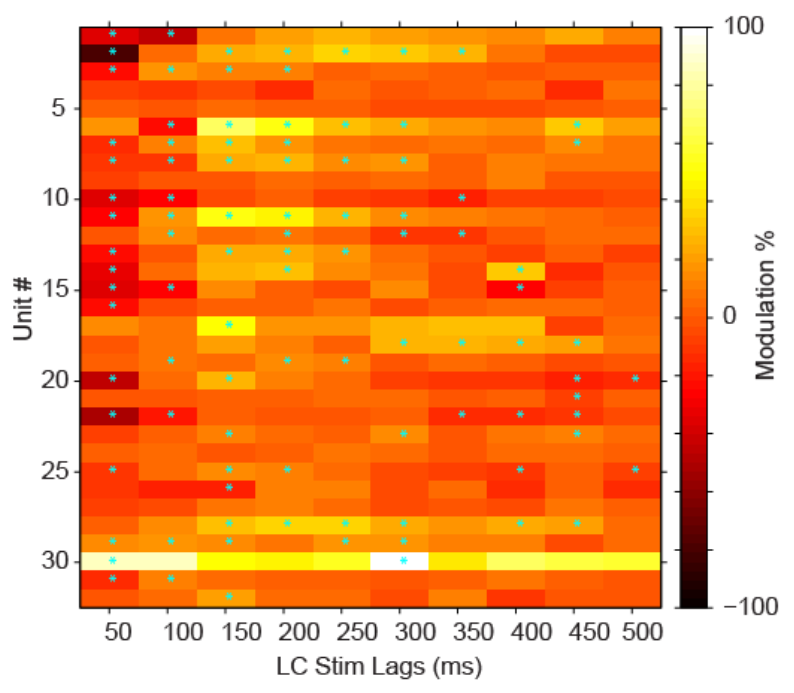

Figure 4. Excitation of BC evoked response by LC micro-stimulation.

(A1) LC was micro-stimulated 150-ms before whisker deflection. LC micro-stimulation current was $100 \mu \mathrm{A}$. (A2) Raster plot of a typical multi-unit in three types of trials: those with whisker deflections but no LC micro-stimulation (brown); those with LC micro-stimulation but no whisker deflections (gray); and those that contained both LC micro-stimulation and whisker deflections (blue). (A3) PSTHs of the typical unit in A2. (A4) Average of normalized PSTHs across all recorded multi-units. Shaded error bars show standard error of mean (SEM) across multiunits. (B) Scatter plot of the BC early response (1-75) during LC micro-stimulation versus no LC 
micro-stimulation condition. Each dot-color shows one unit-current level. Error bars show SEM across trials in each axis. A black arrow (red dot) shows a representative unit in panel $A$. (C) Summary of evoked modulation across units in two current levels; 50 and $100 \mu A(n=32)$. Cyan stars show statistically significant modulations, $p<0.05$, permutation test. 
Figure 5
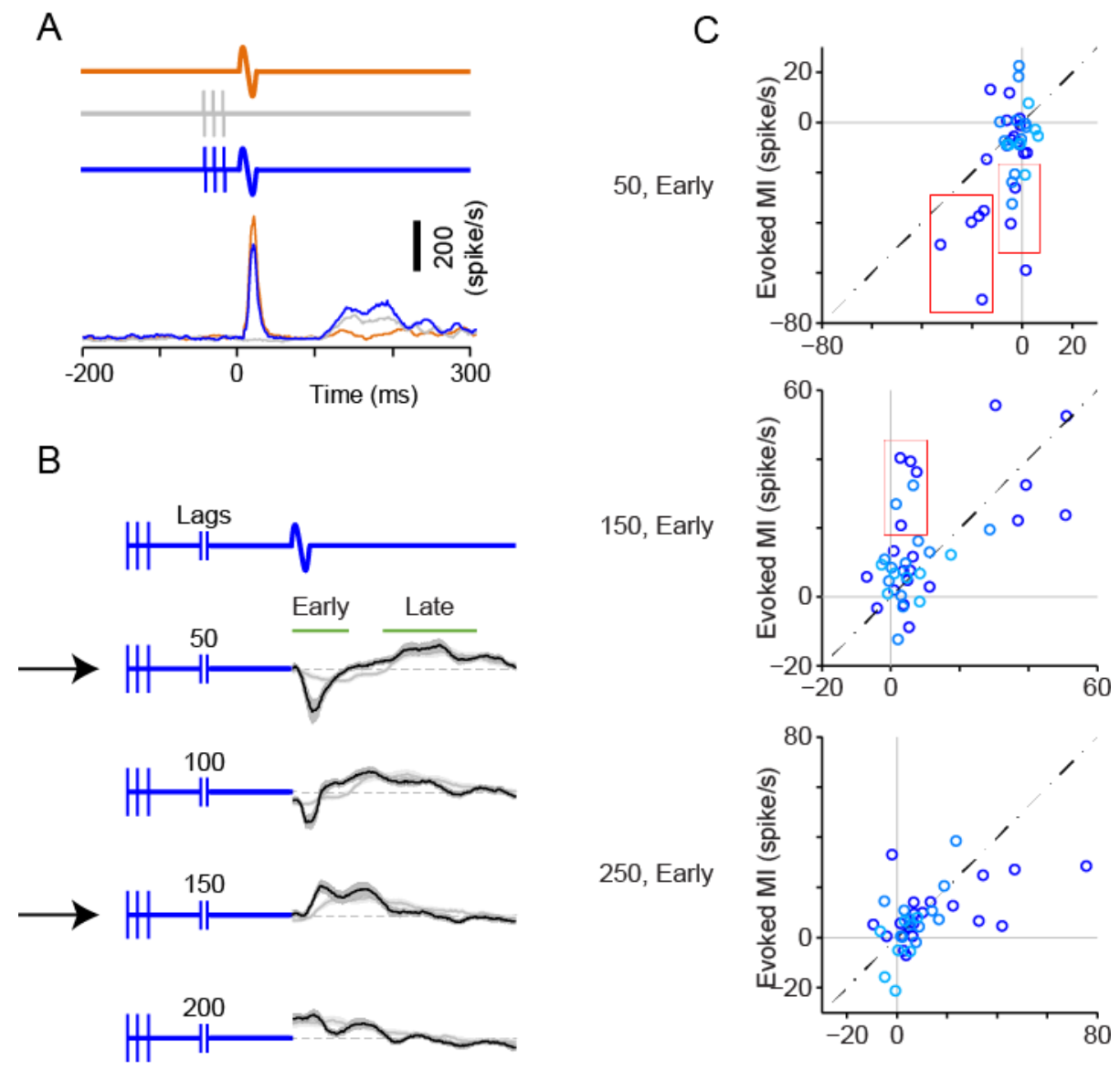

250 , Early
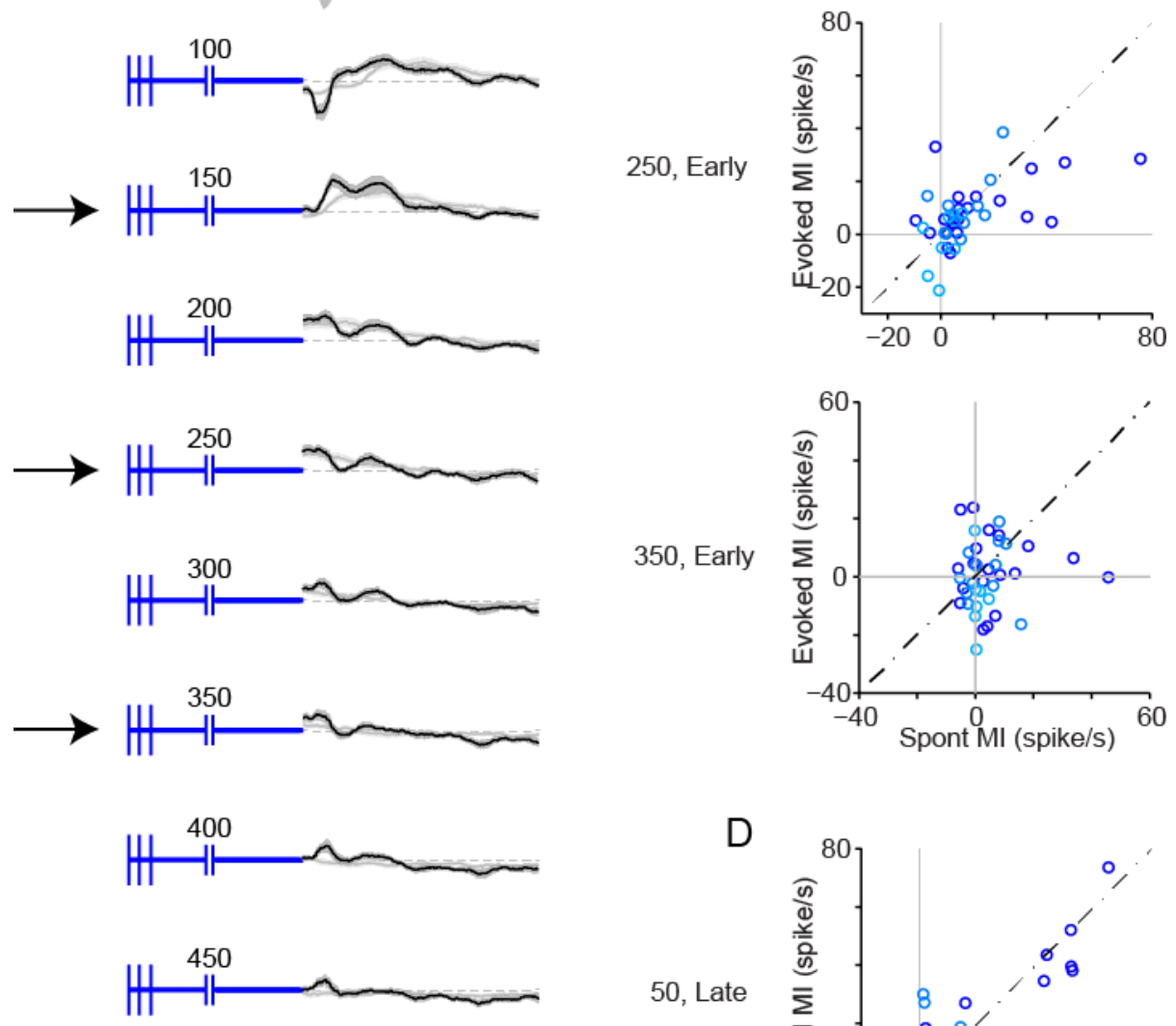

350, Early
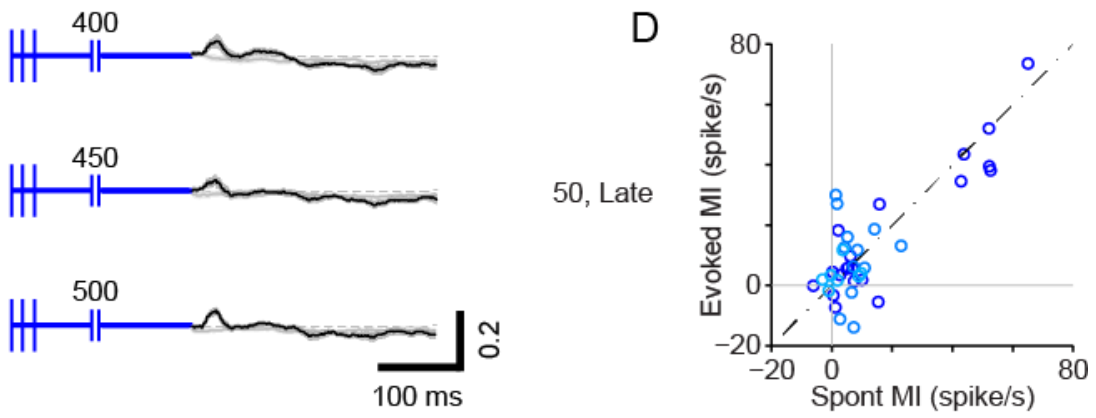


\section{Figure 5. LC micro-stimulation modulation of spontaneous and evoked activity}

(A) PSTH a representative unit. (B) Temporal profile of LC modulation of BC activity;

spontaneous and evoked. Top row shows the time course of LC and whisker stimulation across various trial types. LC micro-stimulation was applied at different time lags from 50 to $500 \mathrm{~ms}$ with a 50-ms step. Gray and black traces show an average of normalized spontaneous and evoked modulation, respectively. Each unit was normalized to peak firing rate in the first $50 \mathrm{~ms}$ after stimulus onset. Spontaneous modulation is the change in activity after LC microstimulation in the absence of any whisker. Evoked modulation is the difference of deflectionevoked PSTHs between LC micro-stimulation and no-LC micro-stimulation trials. The vertical blue lines show the timing of LC micro-stimulation. Dotted lines show 0 point of modulation. Each trace shows the average of modulation across all recorded units for $100 \mu \mathrm{A}$ current level. Three arrows show three lags in panel C. (C) Scatter plots show evoked modulation versus spontaneous modulation for the early response (1:75) in four lags that exhibited the highest modulation. Each dot shows one unit. Dark blue represent $100 \mu \mathrm{A}$ stimulation, light blue represents $50 \mu \mathrm{A}$ stimulation. Dashed lines show unity lines. (D) Same as $C$ but for late response $(125-250)$ at $50 \mathrm{~ms}$ lag. 


\section{Figure 6}

A

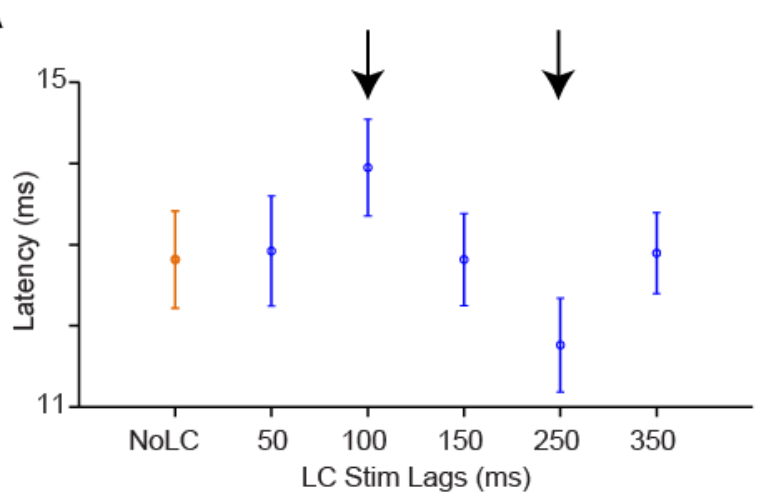

B
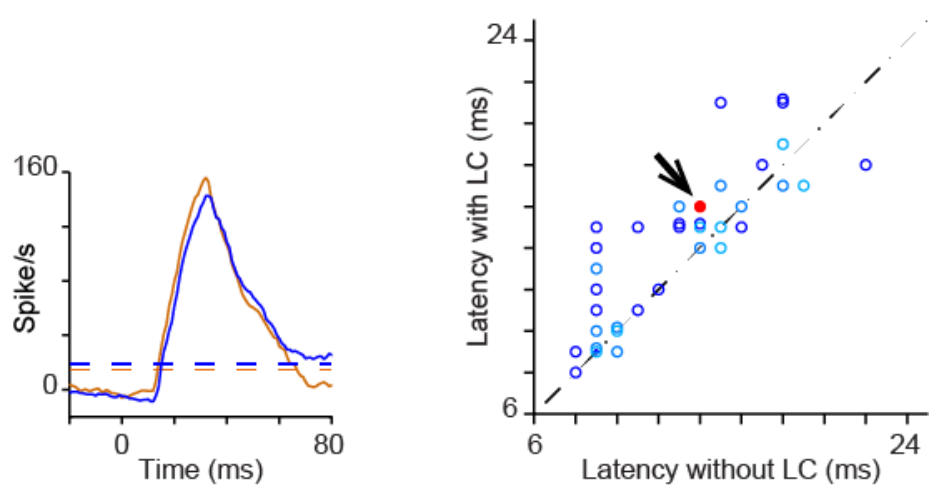

C
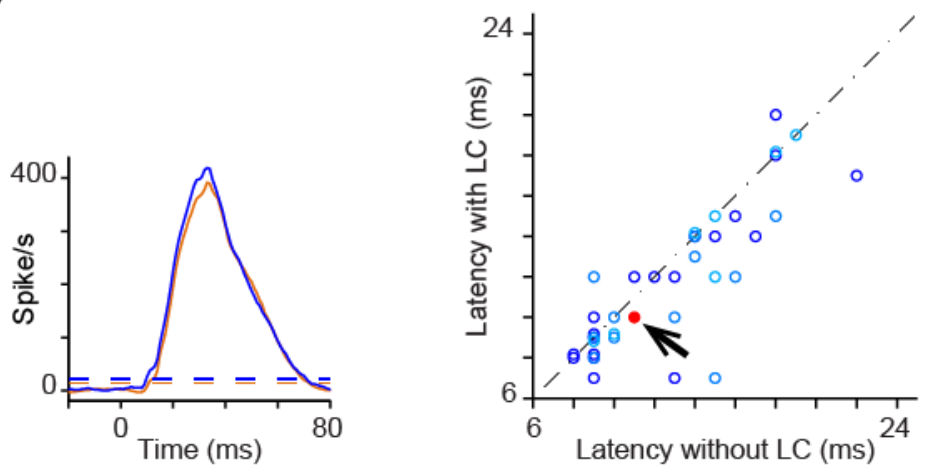

Figure 6. Effect of LC micro-stimulation on response latency.

(A) Average response latency across all units and current levels. At $100 \mathrm{~ms}$ lag, the latency significantly increased and at $250 \mathrm{~ms}$ lag, the latency significantly decreased. (B) Left; PSTHs of a representative multi-unit to whisker deflection of threshold (T) amplitude (bin size, $20 \mathrm{~ms}$; sliding at 1-ms steps). Micro-stimulation was at $100 \mathrm{~ms}$ lag. Right; response latency for all units which showed significant response in two conditions $(n=36)$. Red dots in $B$ and $C$ show a 
representative unit in the left panel. (C) Left; PSTHs of another representative multi-unit. LC micro-stimulation was at 250 ms lag. Right; response latency for all units which showed significant response in two conditions $(n=36)$. 


\section{Figure 7}

A

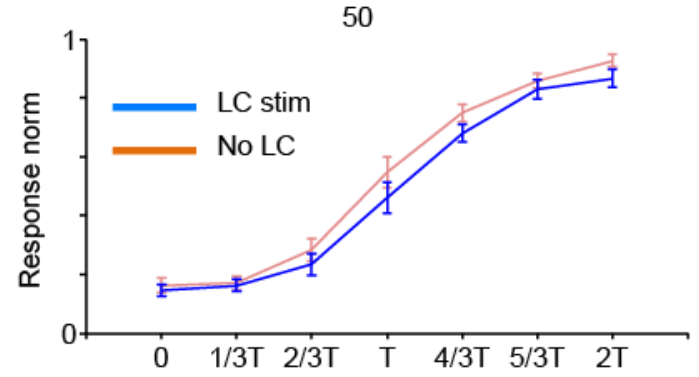

150
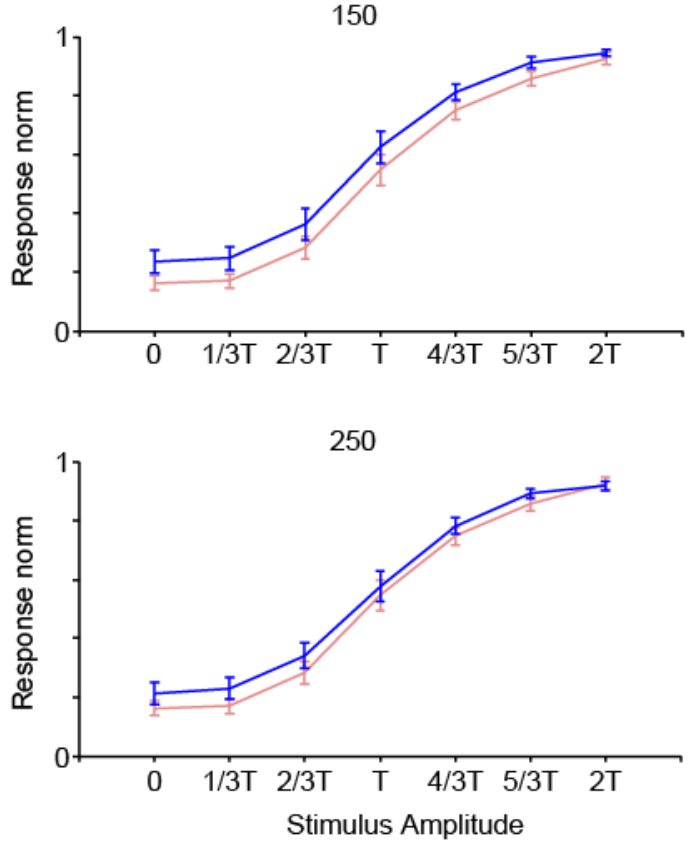

B
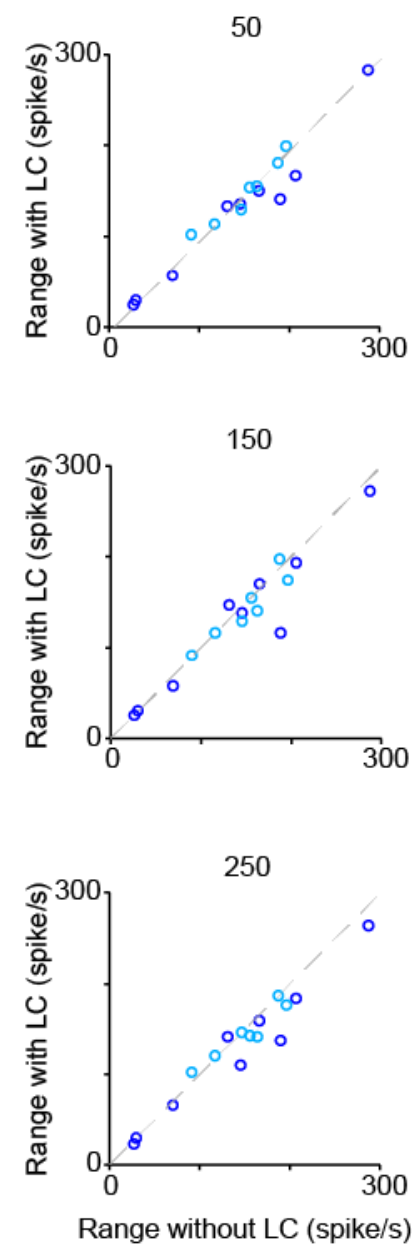

Figure 7. Effect of LC micro-stimulation on stimulus-response function.

(A) The average tuning function across all recorded units at two levels of current (30 and 100 $\mu A)$ in response to the full range of stimulus amplitudes $(n=16)$. (B) Response range calculated as the difference between the highest and lowest response for each unit. Blue and light blue circles show data for $100 \mu \mathrm{A}$ and $30 \mu \mathrm{A}$ respectively. Each panel shows data for three LC microstimulation lags; 50, 150, and 250 ms. Error bars are SEM across units. Spike count has calculated over a 75-ms window post-stimulus onset. 